\title{
A Monte Carlo calculation of the secondary electron emission in the backward direction from a $\mathrm{SiO}_{2}$ macro-capillary
}

\author{
Chao $\mathrm{Li}^{1,2}$, Károly Tókési ${ }^{3, a}$, Luca Repetto ${ }^{4}$, Liye Xiao ${ }^{5}$, Junbiao $\mathrm{Liu}^{5}$, Zhaoshun $\mathrm{Gao}^{5}, \mathrm{Li} \mathrm{Han}^{5}$, Bo $\mathrm{Da}^{6}$, \\ Réka Judit Bereczky ${ }^{3}$, and Zejun Ding ${ }^{1,2}$ \\ 1 Hefei National Laboratory for Physical Sciences at Microscale and Department of Physics, University of Science and Technology \\ of China, Hefei 230026, Anhui, P.R. China \\ 2 Key Laboratory of Strongly-Coupled Quantum Matter Physics, Chinese Academy of Sciences, Hefei 230026, Anhui, P.R. \\ China \\ 3 Institute for Nuclear Research, Hungarian Academy of Sciences (ATOMKI), 4026 Debrecen, Hungary \\ 4 Department of Physics and Nanomed Labs, Università di Genova, Via Dodecaneso 33, 16146 Genova, Italy \\ 5 Institute of Electrical Engineering, Chinese Academy of Sciences, Beijing 100190, P.R. China \\ 6 Center for Materials Research by Information Integration (CMI2), Research and Services Division of Materials Data and \\ Integrated System (MaDIS), National Institute for Materials Science, 1-2-1 Sengen, Tsukuba, Ibaraki 305-0047, Japan
}

Received 27 May 2019 / Received in Final form 20 December 2019

Published online 20 February 2020

(c) The Author(s) 2020. This article is published with open access at Springerlink.com

\begin{abstract}
A Monte Carlo calculation of the secondary electron emission from a $\mathrm{SiO}_{2}$ macro-capillary in the backward direction induced by electron irradiation is presented with the aim to understand transmission guiding of a scanning electron beam through a borosilicate glass macro-capillary. The theoretical modeling of electron transport in $\mathrm{SiO}_{2}$ capillary incorporates the elastic, inelastic and phonon scatterings, resulting, respectively, from the interactions with nucleus, electrons and phonons. The influence on electron inelastic scattering by the insulator bandgap is also considered. In this work a simplified approach has been employed to deal with the charging of the internal wall of the capillary and it is found that at a glancing incident angle, the secondary electrons are mostly originated from the top surface.
\end{abstract}

\section{Introduction}

The pioneering finding of the transmission guiding capability of the polyethylene terephthalate (PET) microcapillary for $3 \mathrm{keV} \mathrm{Ne}{ }^{7+}$ ions [1] started extensive studies of the guiding phenomenon and since then, the transmission guiding for various kinds of slow charged particles has been observed in insulating capillaries made of different materials and sizes [2-7]. The transmission guiding in all these cases irrespective of the experimental differences, originates from the same self-organized charging of the internal wall of the capillary. In guiding experiments, the charged particles are initially forced to collide with the internal wall of the capillary by imposing a tilt angle between the beam and the capillary. With a positive incident charge, when the guiding field is built up, the ions, entering later into the capillary can pass through without charge exchange, indicating that the charged particles never contact the inner surface in their entire motion and are somehow transported toward the exit of the capillary. This process is known as "guiding" in the literature.

However, it is not clear yet whether a negatively charged beam will reproduce such transmission guiding. The first studies on electron guiding through insulating capillaries

\footnotetext{
${ }^{a}$ e-mail: tokesi@atomki.mta.hu
}

show that the electron transmission through capillaries is fundamentally different from the simple picture of positive particles whose guiding is governed solely by Coulomb deflection [6-9]. The major difference can be understood as the impact of a negatively charged particles (mostly electrons) on the surface may lead to a positive charge build-up due to the emission of secondary electrons. Consequently, there may be an attraction of subsequent projectiles towards the capillary wall rather than deflection by the negative Coulomb field. The incident beam with negative charge may also undergo a sequence of elastic and/or inelastic scattering processes at the surface and inside the capillary material resulting in many transmitted particles having suffered energy loss and in the production of secondary electrons which may also reach the capillary exit.

As a supplement to this field, the charging of borosilicate macrocapillaries when a focused electron beam is scanned across its entrance has been studied. The aim of the work was to reveal insight into the mechanisms that can lead to the guiding capabilities of these systems. In particular, preliminary experiments were performed with a scanning electron microscope (SEM) in order to assess the suitability of this instrument to study the guiding capabilities of insulating capillaries, and to analyze the charging of the inner walls and the consequences of the 
(a)

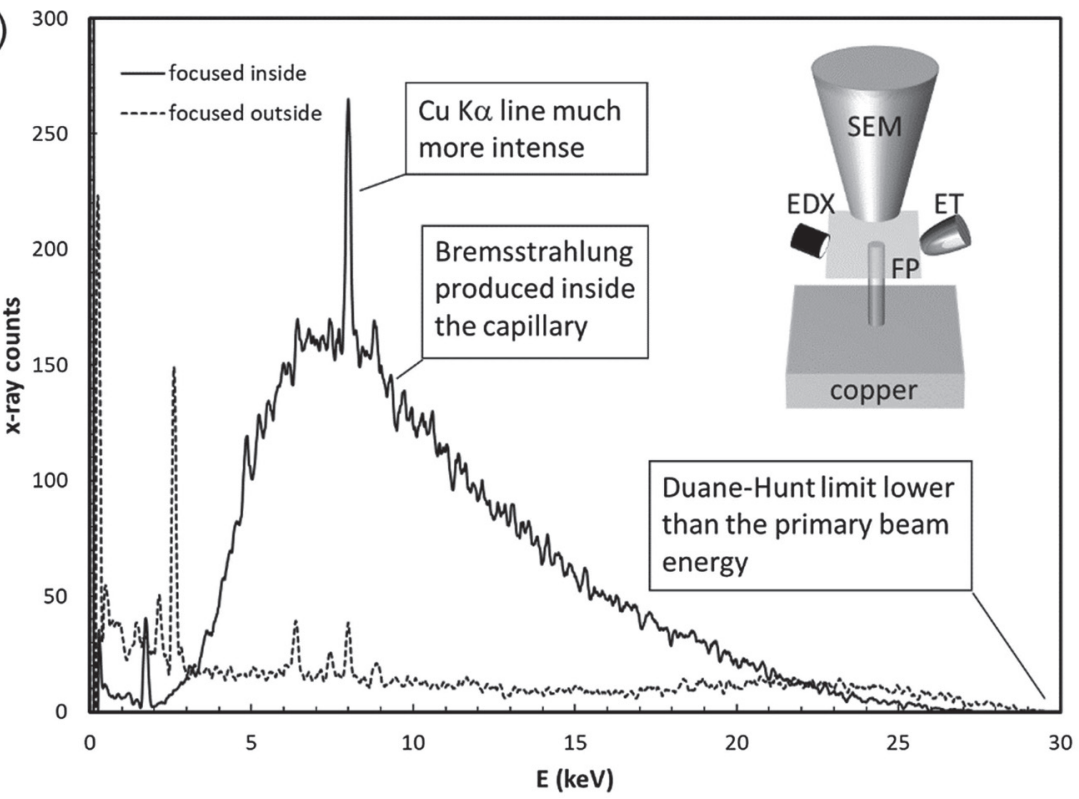

(b)

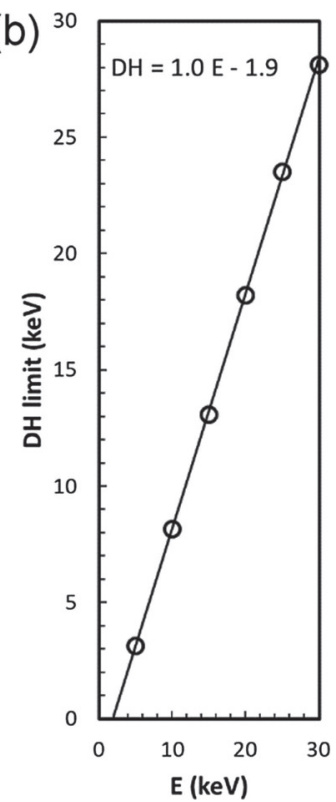

Fig. 1. Indications on the transmission guiding capabilities of the borosilicate capillaries obtained with SEM-EDX. (a) EDX spectra obtained with a $30 \mathrm{keV}$ electron beam focused at the entrance of the capillary (full line) and outside the capillary on the same plane (dashed line) (see the inset schematizing the experiment). The beam travelling inside the capillary produces the expected spectrum of the $\mathrm{Cu}$ target located on the opposite side of the capillary, together with a Bremsstrahlung signal generated by the inelastic interactions with the capillary. The beam travelling outside arrives to the target too defocused to generate the expected spectrum. (b) Analysis of the Duane-Hunt limit in the range 0-30 keV: the electron beam suffers from a constant energy loss of $1.9 \mathrm{keV}$.

charged status on the SEM images for different energies of the primary electron beam in the range between 0.2 and $20 \mathrm{keV}$.

This paper is a preliminary work towards the study on the guiding transmission of the scanning electron beam through the borosilicate glass macro-capillary and uses a Monte Carlo method to reveal the quantitative features of the secondary electron emission from a $\mathrm{SiO}_{2}$ macrocapillary. The Monte Carlo method has been widely and successfully applied as a theoretical tool to the studies on electron-solid interactions [11-14] and thus it is a suitable tool for the present study also. Specifically, the electron transport modeling mainly follows our previous work [15] which studied the electron transport in $\mathrm{SiO}_{2}$ and the charging problems of structures with very complex geometries. The present work is focused on investigating the secondary electron emission from a $\mathrm{SiO}_{2}$ macro-capillary, which has not been previously considered [15]. For such a structure, the entire secondary electron emission consists of the electrons originating not only from the top surface but also from the inner wall. These two distinction components are simulated separately. Furthermore, a simple formula is derived here to describe the actual total electron yield for the capillary.

\section{Experiment}

Indications for properly setting geometries, physical parameters, and approximations for the Monte Carlo simulations, have been obtained by preliminary experiments [10] per- formed with the electron column, the secondary electron detectors (in-lens, and Everhart-Thornley), and the X-ray detector of a Crossbeam 1540 xb (Carl Zeiss AG, Oberkochen, Germany) equipped with an EnergyDispersive X-ray spectroscopy (EDX) unit. The capillary used in the experiment was $12 \mathrm{~mm}$ long with an innerdiameter of $140 \mu \mathrm{m}$ and outer-diameter of $230 \mu \mathrm{m}$. This corresponds to an aspect ratio of about 100 . The potential of this system for investigating the transmission guiding capabilities of borosilicate capillaries for electrons is shown in Figure 1. An electron beam was alternatively focused at the entrance of the capillary and at a point outside the capillary on the same plane (FP-plane shown in the inset of Fig. 1a). X-rays generated in the two cases by the impact of the electrons on a copper target on the opposite side of the capillary were collected by the EDX detector and analyzed. Figure 1 panel a shows the measured spectra where we can see that the capillary maintains the beam at its exit sensibly more intense than the beam that travels outside. The latter reaches the target too defocused to produce the spectrum expected for copper, while the spectrum obtained with the former shows the $\mathrm{Cu}-\mathrm{k} \alpha$ line together the Bremsstrahlung signal produced inside the capillary. The analysis of the Duane-Hunt limit of this Bremsstrahlung in the energy range between 0 and $30 \mathrm{keV}$ (Fig. 1 panel b) allows estimating the energy losses suffered during the transmission in $1.9 \mathrm{keV}$, constant in the explored range.

The inelastic interactions of the beam with the capillary was further investigated by introducing a tilt angle of $2^{\circ}$ to guarantee the initial collision of the primary electrons with the internal wall. The capillary entrance was 

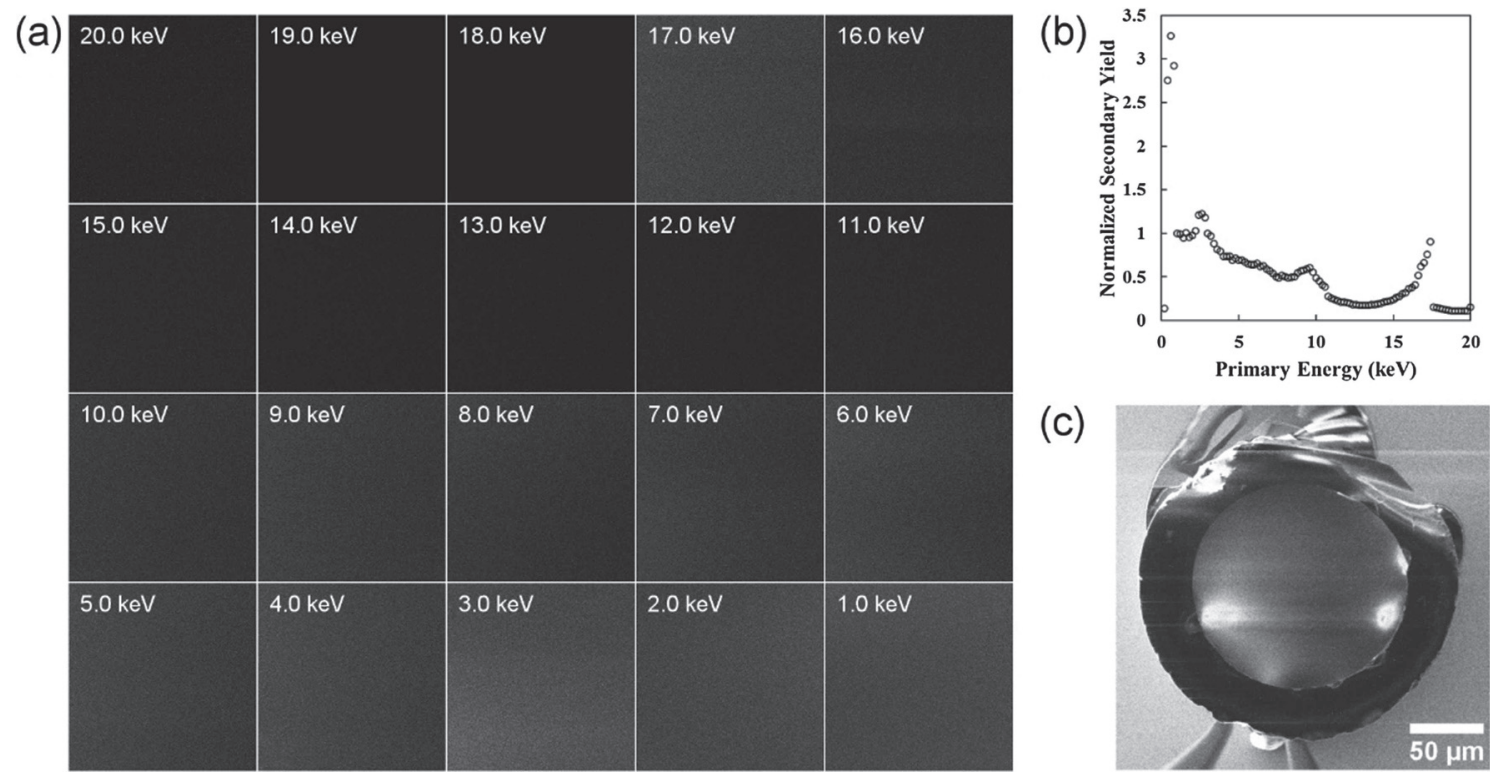

(c)

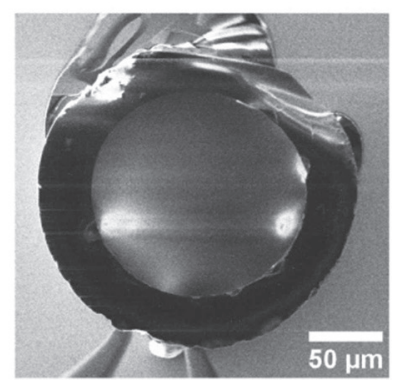

Fig. 2. Preliminary experimental measurements. (a) Sequence of SEM images (in-lens detector) of the capillary entrance for several energies of the primary beam (subset of the full sequence). Each image covers a square-shaped area with side $50 \mu$ mlong. (b) Secondary electron yield as a function of the energy of the primary beam obtained by integrating the images in panel (a). The yield is normalized at $1 \mathrm{keV}$. (c) SEM image of the capillary entrance and its walls at 15 keV (Everhart-Thornley detector) with evidences of charging.

then imaged at varying primary electron energies resulting in a series of SEM images from which the secondary electron yield can be extracted by integrating the signal on the field of view (Figs. 2 panels a and 2b). Charging of the capillary can be deduced by the contrast distortions as shown in Figure 2c. In the case of the SEM test for the macro-capillary, the charging problem is more complicated than those resulting from the irradiation by a static single charged particle beam. Both the point of incidence at the entrance plane and the collision position on the internal wall for the primary electrons vary as functions of time due to the scanning of the primary electron beam. Finally, it is to be noted that the contrast of the images only provides information on the relative intensity of the detected secondary electrons at different primary energy, while an absolute quantification of the secondary electron yield was not possible because of the lack of calibration of the employed detectors.

\section{Theory}

Here, the main points of the theoretical modeling [15], including the elastic, inelastic and phonon scatterings encountered by transporting electrons in an insulating solid sample, are briefly described.

\subsection{Electron elastic scattering}

Electron elastic scattering is described by the Mott cross section [16], which is calculated with the Thomas-FermiDirac atomic potential [17] and describes the probability for the electron to be scattered into a solid angle $d \Omega$ which is located at the polar angle of $\theta$ relative to the previous transport direction,

$$
\frac{d \sigma_{e}}{d \Omega}=|f(\theta)|^{2}+|g(\theta)|^{2},
$$

where the scattering amplitudes,

$$
\begin{aligned}
f(\theta)= & \frac{1}{2 i k} \sum_{\ell=0}^{\infty}\left\{(\ell+1)\left(e^{2 i \delta_{\ell}^{+}}-1\right)\right. \\
& \left.+\ell\left(e^{2 i \delta_{\ell}^{-}}-1\right)\right\} P_{\ell}(\cos \theta) \\
g(\theta)= & \frac{1}{2 i k} \sum_{\ell=1}^{\infty}\left\{-e^{2 i \delta_{\ell}^{+}}+e^{2 i \delta_{\ell}^{-}}\right\} P_{\ell}^{1}(\cos \theta),
\end{aligned}
$$

can be calculated by partial wave expansion method [18]. In equation $(2), P_{\ell}(\cos \theta)$ and $P_{\ell}^{1}(\cos \theta)$ are respectively the Legendre and the first-order associated Legendre functions; $\delta_{\ell}^{+}$and $\delta_{\ell}^{-}$are respectively spin-up and spin-down phase shifts of the $\ell$ th partial wave.

\subsection{Electron inelastic scattering}

The electron inelastic scattering is described by a dielectric functional approach in which the double differential scattering cross section is given by

$$
\frac{d^{2} \lambda_{\text {in }}^{-1}}{d(\hbar \omega) d q}=\frac{1}{\pi a_{0} E} \operatorname{Im}\left\{\frac{-1}{\varepsilon(q, \omega)}\right\} \frac{1}{q},
$$

where $\lambda_{\text {in }}$ is the electron inelastic mean free path (IMFP), $\hbar \omega$ the energy loss, $\hbar q$ the momentum transfer, $a_{0}$ the 
Bohr radius, $E$ the electron energy, and $\varepsilon(q, \omega)$ the dielectric function of the solid. Integrating equation $(3)$ in $(q, \omega)$ space enables the numerical derivation of IMFP and, thus, $\hbar \omega$ and $\theta$ can be sampled. Details of the calculations can be found in our previous work [11]. The energy loss function, $\operatorname{Im}\{-1 / \varepsilon(q, \omega)\}$, is modeled by Lorentz oscillators [19]:

$$
\operatorname{Im}\left\{\frac{-1}{\varepsilon(q, \omega)}\right\}=\operatorname{Im}\left\{\frac{-1}{1+\sum_{j} \chi_{j}(q, \omega)}\right\},
$$

where $\chi_{j}(q, \omega)$ is the complex electronic susceptibility of the $j$ th oscillator,

$$
\chi_{j}(q, \omega)=\Pi_{j}^{2} \frac{f_{j}}{\omega_{j}^{2}(q)-\omega^{2}-i \omega \Gamma_{j}(q)},
$$

where $\Pi_{j}=\sqrt{4 \pi n_{j} e^{2} / m}$ is plasmon frequency, $e$ the electron charge, $m$ the electron mass; $f_{j}, \omega_{j}(q)$ and $\Gamma_{j}(q)$ are oscillator parameters which can be known via the following dispersion relations,

$$
\begin{gathered}
\hbar \omega_{j}(q)=\hbar \omega_{j}(0)+\alpha \hbar^{2} q^{2} / m ; \\
\Gamma_{j}(q)=\Gamma_{j}(0)\left(1+\beta q^{2}\right),
\end{gathered}
$$

where $\alpha$ and $\beta$ are constants for a particular material. For $\mathrm{SiO}_{2} \alpha \approx 0$ and $\beta \approx 6 \AA^{2}$. The values at $q=0$ of these parameters can be obtained by fitting the optical energy loss function with equation (4) to the experimental data $[20,21]$.

For an insulator, the bandgap modifies the energy loss function and it can be written as [22]:

$$
\operatorname{Im}\left\{\frac{-1}{\varepsilon(q, \omega)}\right\}=\operatorname{Im}\left\{\frac{-1}{1+\sum_{j} \chi_{j}(q, \omega)}\right\} \theta\left(\hbar \omega-E_{g}\right),
$$

where $E_{g}=8.9 \mathrm{eV}$ for $\mathrm{SiO}_{2}$.

\subsection{Electron-phonon interaction}

Phonons can be either created or annihilated when electrons are transported in a solid sample. The frequencies for an electron of energy $E$ and effective mass $m^{*}$ to create and annihilate a longitudinal optical (LO) phonon of energy $\hbar \omega, f^{+}$and $f^{-}$, can respectively be given by [23]

$$
\begin{aligned}
& f^{+}=(n+1) \frac{1}{\hbar^{2}} \sqrt{\frac{m^{*}}{2 E}} \frac{e^{2}}{4 \pi \varepsilon_{0}}\left(\frac{1}{\varepsilon_{\infty}}-\frac{1}{\varepsilon}\right) \hbar \omega \\
& \times \ln \frac{1+\sqrt{1-(\hbar \omega / E)}}{1-\sqrt{1-(\hbar \omega / E)}} ; \\
& f^{-}=n \frac{1}{\hbar^{2}} \sqrt{\frac{m^{*}}{2 E}} \frac{e^{2}}{4 \pi \varepsilon_{0}}\left(\frac{1}{\varepsilon_{\infty}}-\frac{1}{\varepsilon}\right) \hbar \omega \ln \frac{1+\sqrt{1+(\hbar \omega / E)}}{-1+\sqrt{1+(\hbar \omega / E)}},
\end{aligned}
$$

where $\varepsilon$ and $\varepsilon_{\infty}$ are respectively the static and optical dielectric constants. For $\mathrm{SiO}_{2}, \varepsilon=3.9$ and $\varepsilon_{\infty}=2.25$.

Thus, the interactions of the transporting electrons in $\mathrm{SiO}_{2}$ of energy less than $30 \mathrm{eV}$ with two LO-phonon modes of the energy of $\hbar \omega_{1}=0.06 \mathrm{eV}$ and $\hbar \omega_{2}=0.153 \mathrm{eV}[15,19]$ are considered through equations (9) and (10).

\subsection{Calculation procedure}

In our simulation, the dielectric function approach is used to describe the electron inelastic scattering, in which the dielectric function of the insulator needs to be known. On the microscopic level, the motion of the bound electrons (those intrinsic to the insulator) relative to the nucleus can be theoretically approximated as the damped harmonic oscillation, which polarizes the atom due to the formation of the dipole between nucleus and electron. In combining the damped harmonic oscillation and the atom polarization, the Lorentz oscillator is used to model the electronic susceptibility $\chi$ in equation (5). Each oscillator has three important parameters, i.e. $f$ (strength), $\omega$ (frequency) and $\Gamma$ (life time). Beside these oscillator parameters, we also need to know how many electrons contribute to the same oscillator, i.e. we have to know the electron concentration $n$. So $n$ electrons oscillate in the same manner, such that their collective motion can be seen as a plasmon, with the frequency given by $\Pi=\sqrt{4 \pi n e^{2} / m}$. The bound electrons could have numerous oscillation modes, requiring the introduction of many Lorentz oscillators. Then, the dielectric function of the insulator can be expressed as $\varepsilon=1+\sum_{j} \chi_{j}$ where the subscript $j$ runs over all the electronic-susceptibility components corresponding to the Lorentz oscillators. With the knowledge of the dielectric function, the electron inelastic scattering can then be described.

In particular, we used 95 oscillators to fit the optical energy loss function of $\mathrm{SiO}_{2}$. The fitting result can be found in reference [15]. During the simulation, the incidence primary electrons start at the entrance of the capillary with the beam current of $0.16 \mathrm{nA}$. The starting positions of the primary electrons are determined by random numbers, and are uniformly distributed at the entrance of the capillary. This is close to the real condition in which the focused electron beam is scanned very quickly at the entrance. The incidence direction is then determined by the angle of the initial electron velocity vector and the central axis of the capillary, which is taken as $2^{\circ}$ (see Fig. 3). After the primary electrons are incident into the hole of the capillary, it is possible for them to hit the surface of the inner wall, whereas the specific hitting position will be different from the intersection between the initial incidence direction of the primary electron with the surface of the inner wall, because the influence of charging on electron trajectory are taken into account. Moreover, the duration of the primary electron irradiation on the capillary is taken as $40 \mathrm{~ms}$ with the total number of the incident electrons of $4 \times 10^{7}$.

\section{Results and discussion}

Focus now switches to the secondary electron emission from the $\mathrm{SiO}_{2}$ macro-capillary which, having the same size as that of the borosilicate glass macro-capillary used in the experiment. The emission is induced by the irradiation of an electron beam scanning across the entrance with a 


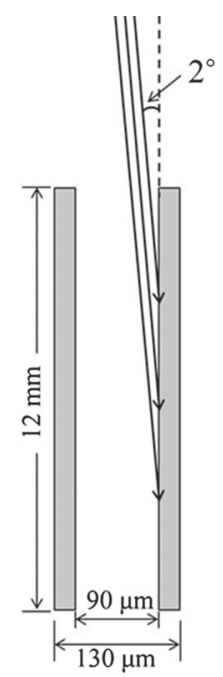

Fig. 3. The schematic graph of the irradiation of the primary electrons on the internal wall of the $\mathrm{SiO}_{2}$ macro-capillary with the tilt angle of $2^{\circ}$ between the axis and the electron incident direction. The capillary has the length of $12 \mathrm{~mm}$, the innerdiameter of $90 \mu \mathrm{m}$ and the outer-diameter of $130 \mu \mathrm{m}$, and hence the aspect ratio of about 100 .

tilt angle of $2^{\circ}$. During the scanning, the internal wall and also the top surface suffer electron irradiation, so the total secondary electron emission from the capillary can be seen as the summation of that originating respectively from these two parts. The secondary electron yield of the capillary can be simply estimated by

$$
\begin{aligned}
\delta & =\delta_{\text {wall }} \cdot \pi r_{1}^{2} / \pi r_{2}^{2}+\delta_{\text {top }} \cdot\left(\pi r_{2}^{2}-\pi r_{1}^{2}\right) / \pi r_{2}^{2} \\
& =\delta_{\text {wall }} \times 0.47+\delta_{\text {top }} \times 0.53,
\end{aligned}
$$

where $\delta_{\text {wall }}$ and $\delta_{\text {top }}$ are respectively the secondary electron yields of the internal wall and the top surface. In our case $r_{1}=45 \mu \mathrm{m}$ and $r_{2}=65 \mu \mathrm{m}$ are the inner and outerradiuses of the capillary respectively (see the schematic graph of the capillary entrance in Fig. 3).

The initial imaging was of the macro-capillary, whose shape and size has been previously stated, by using a SEM. In the imaging, the primary electron beam irradiates the capillary entrance almost vertically (only with a tilt angle of $2^{\circ}$ relative to the capillary axis), as shown in Figure 3. For the SEM images obtained at different primary energies, the image contrasts are distorted and differ each other. A common feature, irrespective of the energy dependence of the image contrast, amongst all the SEM images, is that the contrast is symmetrical relative to the dash line shown in Figure 4. Note that this dash line is only an auxiliary line and has no practical meaning; meanwhile, its position and orientation in Figure 2c are exactly the same as in Figure 4. This suggests the trapped charges resulting from the primary electron irradiation are also distributed symmetrically relative to this line - the left-wall and the right-wall share the same charge distribution.

Another problem for the incident primary electron beam is that it only has the momentum components

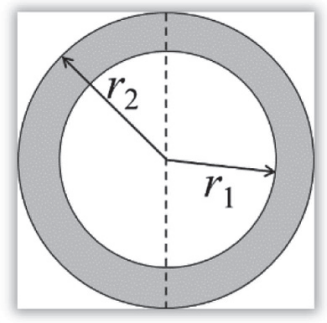

Fig. 4. Schematic graph of the cross section of the $\mathrm{SiO}_{2}$ macrocapillary which is perpendicular to the capillary axis.

parallel with the incidence plane, one is along the capillary axis and the other along the surface normal of the inner wall (Fig. 3). Due to the very limited tilt angle of $2^{\circ}$ and the keV energy scale of primary electrons, the primary electron beam's momentum component along the surface normal of the inner wall will be rather limited, such that the maximum depth penetrated into the wall for the primary electrons will be quite small. Based on these practical situations, line-shape charge distributions are assumed for both the left-wall and the right-wall.

The large aspect ratio of the capillary means that the region of the top circular entrance will not allow many secondary electrons to escape from the capillary hole successfully. Here, it was first assumed that the amount of the secondary electrons escaping from the capillary hole through the entrance is negligible compared with that of the primary electrons incident into the capillary through the entrance, such that the primary electrons incident into the inner wall will mostly be trapped. Therefore, the amount of primary electrons penetrated into the right-wall is saved for different sites and half of the saved penetration-amount distribution was used as the line-shape trapped charge distribution for both the rightwall and the left-wall.

Furthermore, the line-shape trapped charges, which are located on the surfaces of the left-wall and the right-wall, can further be seen as located on two parallel foils, with which the electric field distribution can be obtained by the mono-image charge method, as described in our previous work [15]. Under such an electric field, the flight of the secondary electrons emitted from the inner wall is tracked within a cylindrical geometry having the same size as the capillary hole with only those passing through the top circular entrance considered as effective signals. Tracking is terminated for particles which collide with the inner wall or particles which break the cylindrical geometry. Such collisions may broaden the charge distribution perpendicular to the incidence plane. However, considering the macroscopic size of the capillary, the line-shape charge distribution mentioned above was still used. The influence of the electric field on the energy and the trajectory of the incident primary electrons have also been taken into account in the same way as that for the secondary electrons once the primary electrons have penetrated the hole.

Figure 5 shows the simulated $\delta_{\text {wall }}$ where charging has been considered as described above. $\delta_{\text {wall }}$ is very small compared with the yields of bulk materials which usually reach the order of the unity $[24,25]$. This is closely 


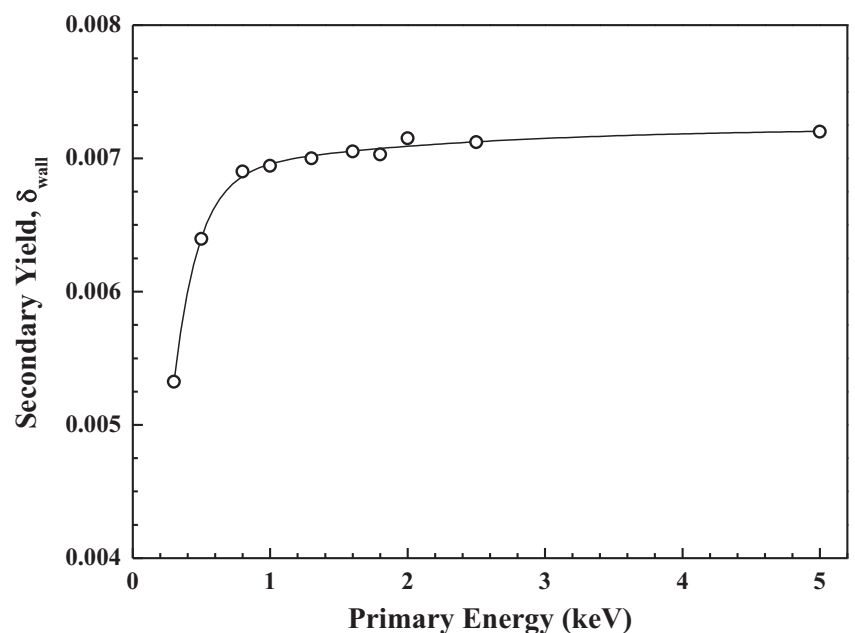

Fig. 5. The simulated secondary electron yields of the internal wall of the capillary where the charging has been considered by using the simplified approach with the current intensity of $0.16 \mathrm{nA}$ in the simulation.

related to the small tilt angle and the large aspect ratio, such that the former factor enables the primary electrons enters deeply into the hole while the latter allows a limited vertical range for secondary electrons to be emitted from entrance hole.

Due to the incorporation of charging, the secondary electron yield becomes time-dependent. Figure 6 shows the evolution of the secondary electron yield of the inner wall of the capillary as a function of time. We note that the secondary electron yield becomes saturated at about $40 \mathrm{~ms}$, which can also be found in other primary electron energies. The secondary electron yield shown in Figure 5 counts the secondary electron emission from $t=0$ to $t=40 \mathrm{~ms}$ for all the cases, which is to comply with the principle of SEM imaging that all the emitted secondary electrons would contribute to the image before it is finally shown on the screen.

On the other hand, in modern SEMs, the beam current could range from $\mathrm{pA}$ to $\mu \mathrm{A}$, and the use of the beam current of $0.16 \mathrm{nA}$ is very normal. In addition, before the steady state is reached, not only charging but also its influence on secondary electron emission is always varying. In order to incorporate the influence of the charging on secondary electron emission more accurately, the simulation regularly updates the charging status many times throughout the entire primary electron irradiation process. Specifically, we consider a short time duration $\Delta t$ within which only a small number of primary electrons are incident into the capillary: within the first $\Delta t$, the influence of charging on secondary electron emission is neglected, and at the end of the first $\Delta t$, the charging status can be obtained according to the primary electron irradiation in the $\Delta t$; within the second $\Delta t$, the influence of charging (the charging status is taken as that obtained at the end of the first $\Delta t$ ) on secondary electron emission is taken into account, and the charging status at the end of the second $\Delta t$ can be obtained according to the primary electron irradiation in these two short time durations. In

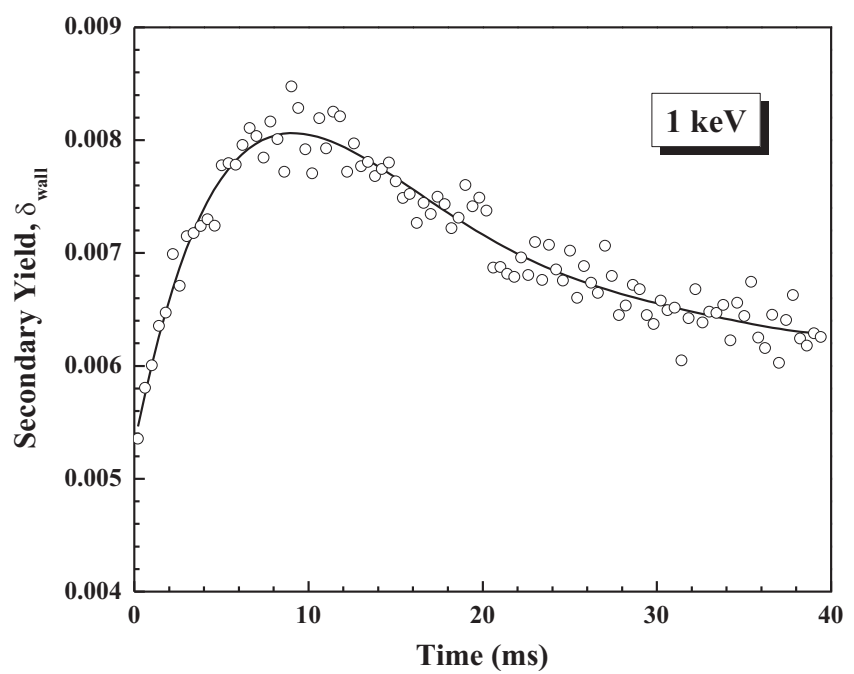

Fig. 6. The evolution of the secondary electron yield of the inner wall of the capillary, emitted in the backward direction, as a function of the primary electron irradiation time.

Figure 6, the charging status is always taken from the previous primary electron irradiation. In such a manner, the simulation is pushed forward until the total simulation time reaches the required one.

We found a strong enhancement in the secondary electron emission near the capillary edge for the top surface and this is comparable to the line scan profile of a trapezoidal line structure [26-28]. However, it is necessary to mention that the difference between the outer and inner radiuses of the capillary, i.e. $r_{2}-r_{1}$, is still in the order of $\mu \mathrm{m}$, which guarantees the majority of secondary electron emission will not be influenced by the capillary edge. In addition, the tilt angle of as small as $2^{\circ}$ is negligible for the secondary electron emission from the top surface. Therefore, $\delta_{\text {top }}$ can be represented by the secondary electron yield of a bulk sample induced by the vertical electron irradiation. Figure 6 shows the simulated secondary electron yield as a function of the primary electron energy, where the primary electrons vertically irradiate a $\mathrm{SiO}_{2}$ bulk without taking charging into account.

According to equation (11), however, $\delta_{\text {top }}$ dominates the secondary electron emission for the $\mathrm{SiO}_{2}$ macro-capillary. This conclusion is obtained by the simple comparison of the results shown in Figures 5 and 7. The linear transformation of the image contrast into the secondary electron yield shows that the variation tendency of $\delta_{\text {top }}$ as a function of the primary electron energy (Fig. 7) qualitatively agrees with the SEM images $[10,28]$. This suggests that the present analysis is reasonable but due to the complexity of the present problem, this work is only a preliminary consideration and more rigorous analysis from a theoretical perspective is still needed.

\section{Conclusions}

A Monte Carlo method has been applied to the simulation of the secondary electron emission from a $\mathrm{SiO}_{2}$ 


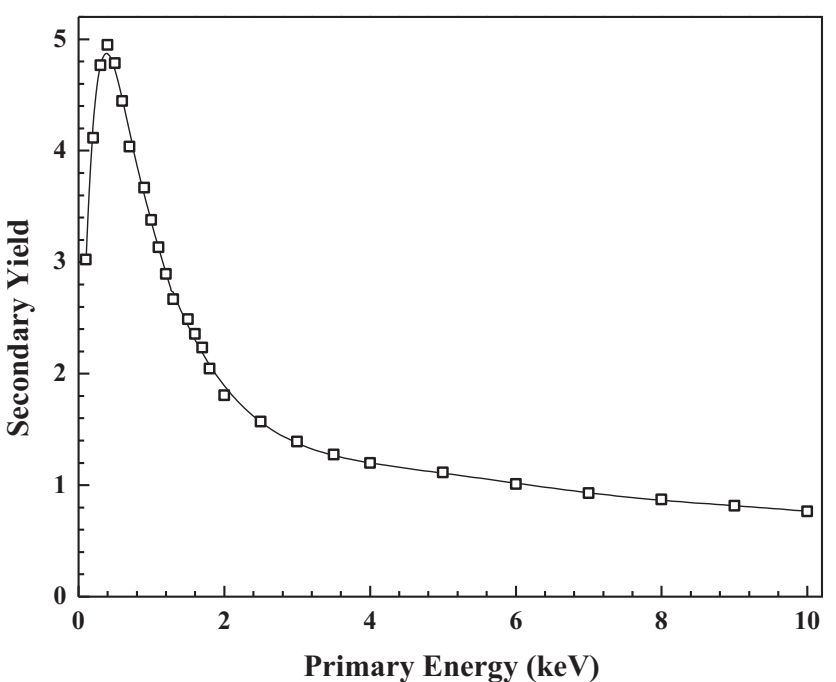

Fig. 7. The simulated secondary electron yields of a $\mathrm{SiO}_{2}$ bulk under the vertical irradiation of the primary electrons, where charging has not been considered.

macro-capillary due to electron irradiation. For the analysis of the present charging problem, a simplified approach was employed to deal with the charging of internal wall of the capillary. These calculations on the secondary electron yields of the internal wall and the top surface found that, with a small tilt angle of $2^{\circ}$, the top surface that dominates the secondary electron emission for the $\mathrm{SiO}_{2}$ macrocapillary.

Open access funding provided by MTA Institute for Nuclear Research (MTA ATOMKI). The work was supported by the National Natural Science Foundation of China (No. 11574289), National Key Research and Development Project (2019YFF0216404), Chinese Education Ministry through "111 Project 2.0" (BP0719016), National Research, Development and Innovation Office (NKFIH) under grant KH126886, and the European Cost Actions CA15107 (MultiComp). We are thankful to Dr. Andrew Cheesman for the critical reading of the manuscript. We thank supercomputing center of USTC for the support of parallel computing.

Open Access This is an open access article distributed under the terms of the Creative Commons Attribution License (http://creativecommons.org/licenses/by/4.0/), which permits unrestricted use, distribution, and reproduction in any medium, provided the original work is properly cited.

\section{Author contribution statement}

All authors discussed the results and contributed to the final manuscript. C. Li performed the calculations and analyzed the results. L. Repetto performed the systematic experiments and evaluated the experimental observations. R.J. Bereczky participated in the performance of the measurements, K. Tökési and Z. J. Ding defined the model and interpreted the simulation results.

\section{References}

1. N. Stolterfoht, J.H. Bremer, V. Hoffmann, R. Hellhammer, D. Fink, A. Petrov, B. Sulik, Phys. Rev. Lett. 88, 133201 (2002)

2. R.J. Bereczky, G. Kowarik, F. Aumayr, K. Tökési, Nucl. Instrum. Methods Phys. Res. B 267, 317 (2009)

3. G. Kowarik, R.J. Bereczky, F. Aumayr, K. Tökési, Nucl. Instrum. Methods Phys. Res. B 267, 2277 (2009)

4. T.M. Kojima, T. Ikeda, Y. Kanai, Y. Yamazaki, Nucl. Instrum. Methods Phys. Res. B 354, 16 (2015)

5. E. Gruber, G. Kowarik, F. Ladinig, J.P. Waclawek, D. Schrempf, F. Aumayr, R.J. Bereczky, K. Tőkési, P. Gunacker, T. Schweigler, C. Lemell, J. Burgdörfer, Phys. Rev. A 86, 062901 (2012)

6. B.S. Dassanayake, S. Das, R.J. Bereczky, K. Tőkési, J.A. Tanis, Phys. Rev. A 81, 020701(R) (2010)

7. A.R. Milosavljević, K. Schiessl, C. Lemell, K. Tőkési, M. Mátéfi-Tempfli, S. Mátefi-Témpfli, B.P. Marinković, J. Burgdörfer, Nucl. Instrum. Methods Phys. Res. B 279, 190 (2012)

8. A.R. Milosavlević, J. Jureta, Gy. Vikor, Z.D. Pešić, D. Šević, M. Matefli-Tempfli, S. Matefli-Tempfli, B.P. Marinković, Europhys. Lett. 86, 23001 (2009)

9. K. Schiessl, K. Tokesi, B. Solleder, C. Lemell, J. Burgdorfer, Phys. Rev. Lett. 102, 163201 (2009)

10. R.J. Bereczky, L. Repetto, U. Valbusa, K. Tökési, Charging of borosilicate capillaries: hysteresis and border effects, in International Conference on Atomic Collisions in Solids, 2014, Debrecen, Hungary, 2014

11. Z.J. Ding, R. Shimizu, Scanning 18, 92 (1996)

12. S.F. Mao, Y.G. Li, R.G. Zeng, Z.J. Ding, J. Appl. Phys. 104, 114907 (2008)

13. Y.G. Li, Z.J. Ding, Z.M. Zhang, J. Appl. Phys. 106, $024316(2009)$

14. H. Xu, B. Da, J. Tóth, K. Tőkési, Z.J. Ding, Phys. Rev. B 95, 195417 (2017)

15. C. Li, S.F. Mao, Y.B. Zou, Y.G. Li, P. Zhang, H.M. Li, Z.J. Ding, J. Phys. D: Appl. Phys. 51, 165301 (2018)

16. N.F. Mott, Proc. R. Soc. A 124, 425 (1929)

17. R.A. Bonham, T.G. Strand, J. Chem. Phys. 39, 2200 (1963)

18. Y. Yamazaki, Ph.D. thesis, Osaka University, 1977

19. J.C. Kuhr, H.-J. Fitting, J. Electron. Spectrosc. Relat. Phenom. 105, 257 (1999)

20. E.D. Palik, Handbook of Optical Constants of Solids (Academic, Orlando, 1985)

21. Y. Sun, H. Xu, B. Da, S.F. Mao, Z.J. Ding, Chin. J. Chem. Phys. 29, 663 (2016)

22. D. Tahir, H.L. Kwon, H.C. Shin, S.K. Oh, H.J. Kang, S. Heo, J.G. Chung, J.C. Lee, S. Tougaard, J. Phys. D: Appl. Phys. 43, 255301 (2010)

23. H.-J. Fitting, J.U. Friemann, Phys. Status Solidi A 69, 349 (1982)

24. H. Seiler, J. Appl. Phys. 54, R1 (1983)

25. C.G. Walker, M.M. El-Gomati, A.M. Assa'd, M. Zadrazil, Scanning 30, 365 (2008)

26. Y.G. Li, S.F. Mao, H.M. Li, S.M. Xiao, Z.J. Ding, J. Appl. Phys. 104, 064901 (2008)

27. Y.G. Li, P. Zhang, Z.J. Ding, Scanning 35, 127 (2013)

28. Y.B. Zou, M.S.S. Khan, H.M. Li, Y.G. Li, W. Li, S.T. Gao, L.S. Liu, Z.J. Ding, Measurement 123, 150 (2018)

29. L. Repetto et al. (2019) to be published 